\title{
Correlação entre independência funcional e qualidade de vida de pacientes com esclerose lateral amiotrófica
}

\author{
Nathalia Priscilla Oliveira Silva ${ }^{a}$, Lizianne Juline do Nascimento e Silva Martins $s^{b}$, \\ Thaiana Barbosa Ferreira ${ }^{c}$, Fabrícia Azevedo da Costa Cavalcanti ${ }^{\mathrm{d}}$ \\ aPrograma de Pós-graduação em Fisioterapia, \\ Universidade Federal do Rio Grande do Norte - UFRN, Natal, RN, Brasil \\ bUniversidade Federal do Rio Grande do Norte - UFRN, Natal, RN, Brasil \\ 'Faculdade de Ciências da Saúde do Trairí - FACISA, Santa Cruz, RN, Brasil \\ ${ }^{d}$ Departamento de Fisioterapia, Universidade Federal do Rio Grande do Norte - UFRN, Natal, RN, Brasil
}

\begin{abstract}
Resumo: A independência funcional e qualidade de vida das pessoas sofrem um impacto quando elas são acometidas pela esclerose lateral amiotrófica (ELA), doença progressiva e degenerativa. O objetivo do estudo foi investigar a independência funcional e a qualidade de vida de pacientes com ELA em Natal, RN. Realizou-se uma pesquisa transversal com 24 pacientes. Utilizou-se como instrumentos o Amyotrophic Lateral Sclerosis Assessment Questionnaire (ALSAQ-40/BR) e a Medida de Independência Funcional (MIF). Os dados foram analisados através do teste de correlação de Spearman e Mann Whitney. Os indivíduos apresentaram dependência funcional modificada na MIF com média de 64,9 $\pm 20,5$ e tiveram todos os domínios da ALSAQ-40/BR afetados. Houve correlação inversa significativa entre a MIF e os domínios da ALSAQ-40/BR: Mobilidade $(p<0,01)$, AVDs $(p<0,01)$, Alimentação $(p=0,02)$ e Comunicação $(\mathrm{p}<0,01)$, com exceção do Aspecto Emocional. Apesar do número amostral reduzido, todos os pacientes apresentaram diminuição na independência funcional e qualidade de vida. O uso desses instrumentos pode ser uma ferramenta de ajuda na elaboração de planos de intervenção e tratamento interdisciplinar, contribuindo para retardar a dependência funcional e melhorar a qualidade de vida dessas pessoas.
\end{abstract}

Palavras-chave: Esclerose Amiotrófica Lateral, Funcionalidade, Qualidade de Vida.

\section{Correlation between functional independence and quality of life of patients with amyotrophic lateral sclerosis}

\begin{abstract}
Functional independence and quality of life are impacted by amyotrophic lateral sclerosis (ALS), a degenerative and progressive disease. The aim of this study was to investigate the functional independence and quality of life of patients with ALS in the municipality of Natal, Rio Grande do Norte state, Brazil. This is a cross-sectional observational study conducted with 24 patients. The Amyotrophic Lateral Sclerosis Assessment Questionnaire (ALSAQ-40/BR) and the Functional Independence Measure (FIM) were used as evaluation instruments. The data were analyzed through the Spearman's correlation and Mann-Whitney tests. The individuals investigated presented modified functional dependence in the FIM, with mean of $64.9 \pm 20.5$, and alteration in all areas of the ALSAQ-40/ BR. There was significant inverse correlation between FIM and the ALSAQ-40/BR areas of "Mobility" $(p<0.01)$, “Activities of Daily Living (DLAs)" ( $p<0.01)$, "Eating ability" ( $p=0.02)$, and "Communication" $(p<0.01)$, but not in the domain of "Emotional Aspect". Despite the reduced sample, all patients presented reduction in functional
\end{abstract}

Autor para correspondência: Nathalia Priscilla Oliveira Silva, Rua Poço Branco 186, Nova Parnamirim, CEP 59158-280, Parnamirim, RN, Brasil, e-mail: fisio.nathalia@hotmail.com

Recebido em 17/5/2013; Revisão em 6/9/2013; Aceito em 18/3/2014. 
independence and quality of life. The use of these instruments may be a tool to assist the elaboration of intervention plans and interdisciplinary treatment, contributing to retard functional dependence and improve the quality of life of these patients.

Keywords: Amyotrophic Lateral Sclerosis, Functionality, Quality of Life.

\section{Introdução}

A Esclerose Lateral Amiotrófica (ELA) é uma doença degenerativa dos neurônios motores superiores e inferiores que envolve o sistema motor em níveis bulbar, cervical, torácico e lombar; é progressiva e fatal, de causas ainda pouco conhecidas (MITCHELL; BORASIO, 2007). O acometimento dos neurônios motores superiores leva a espasticidade, clônus e reflexos tendinosos hiperativos, enquanto a alteração dos neurônios motores inferiores resulta em fasciculação, atrofia, fraqueza, hiporreflexia, hipotonia e câibras musculares. Além disso, há os sinais bulbares, incluindo disartria, disfagia, sialorréia e labilidade emocional (DURÁN, 2006; NORDON; ESPÓSITO, 2009).

As doenças do neurônio motor estáo numa classe de enfermidade que altera o controle muscular, o que provoca alterações na função e bem-estar daqueles com esse diagnóstico (JENKINSON et al., 1999). Portanto, traz implicaçóes negativas na percepção do estado de saúde e impacto na qualidade de vida (QV). De acordo com a Organizaçáo Mundial da Saúde (OMS), a expressão "qualidade de vida" refere-se à percepção, do indivíduo, da sua posição na vida, dentro do contexto de cultura e sistema de valores e em relaçáo estabelecida com o ambiente em que vive (MINAYO; MARTZ; BUSS, 2000; DINIZ, 2006). A qualidade de vida está relacionada aos aspectos físicos e psicológicos do indivíduo, ao grau de independência, às relaçóes sociais, ao meio ambiente, à religião, à qualidade de vida global e a percepçôes gerais de saúde (FLECK et al, 2008).

A independência funcional está ligada às atividades de vida diária, ou seja, às atividades funcionais que são aquelas identificadas pelo indivíduo como essenciais para a manutenção do seu bem-estar físico e psicológico, assim como para a criaçáo de um senso pessoal de vida significativa (HAMILTON et al., 1993; McNAUGHTON; WEATHERALL; MCPHERSON, 2005). Para alcançar a independência funcional, o indivíduo necessita da integração de recursos cognitivos, comportamentais e sensório-motores, a fim de que as habilidades motoras sejam produzidas de forma efetiva (CESÁRIO; PENASSO; OLIVEIRA, 2006).
Não existe cura para a ELA e em muitos países os indivíduos acometidos por essa doença são acompanhados em centros de referência terciários, que se baseiam nos cuidados interdisciplinares. Nesse sentido, muitos estudos vêm demonstrando que os indivíduos com ELA acompanhados por equipe interdisciplinar em centros de referência apresentam melhor prognóstico (VAN DEN BERG, 2005). No entanto, pouco se sabe acerca do impacto que a evolução da doença ocasiona na qualidade de vida das pessoas acometidas pela ELA. Dessa forma, devido ao comprometimento motor progressivo à medida que a doença evolui, a independência funcional e a qualidade de vida dos pacientes acometidos são itens de fundamental importância e devem ser amplamente estudados em pesquisas acerca da clínica e da epidemiologia da doença.

O presente trabalho objetiva investigar o nível de independência funcional e a qualidade de vida de pacientes diagnosticados com Esclerose Lateral Amiotrófica (ELA) que são acompanhados pelo Centro de Referência em Doença do Neurônio Motor/ELA do Hospital Universitário Onofre Lopes (HUOL), na cidade de Natal, Rio Grande do Norte, Brasil.

\section{Materiais e métodos}

Realizou-se uma pesquisa transversal da qual participaram 24 pacientes acompanhados no Centro de Referência em Doença do Neurônio Motor/ELA do HUOL, na cidade de Natal, Rio Grande do Norte. A amostra foi selecionada por conveniência no período compreendido entre setembro de 2010 e abril de 2011. Foram utilizados como critérios de inclusão o diagnóstico médico provável, possível e definido de ELA, baseado nos critérios utilizados na prática clínica médica de El Escorial revisados em 2000 (BROOKS et al., 2000); os diversos estágios da ELA; o acompanhamento no referido centro. Foram utilizados como critérios de exclusão a presença de patologias associadas que pudessem acarretar sequelas cognitivas e funcionais além das provocadas pela ELA, tais como amputaçáo e doenças crônicas severas (AVC, Parkinson, Alzheimer etc.); acometimento motor severo que impedisse o paciente de responder 
aos protocolos utilizados. Não houve a exclusão de nenhum paciente nesta pesquisa.

O estudo foi aprovado pelo Comitê de Ética em Pesquisa da Universidade Federal do Rio Grande do Norte (Parecer n. 167/2010) e todos os pacientes ou responsáveis assinaram o termo de consentimento livre e esclarecido, autorizando a participação no estudo.

\subsection{Procedimentos}

As coletas foram realizadas no HUOL-UFRN, uma vez por semana, por duas examinadoras previamente treinadas. Inicialmente, os participantes foram identificados por meio de uma ficha de avaliação fisioterapêutica para coletar dados clínicos e pessoais conforme modelo utilizado no Serviço de Fisioterapia do HUOL, contendo a identificação completa do paciente, história da doença atual (HDA), exame clínico geral, antecedentes pessoais e familiares, hábitos de vida (fatores de risco) e medicamentos em uso.

Como instrumento de avaliaçáo da qualidade de vida foi utilizado o Amyotrophic Lateral Sclerosis Assessment Questionnaire (ALSAQ-40/BR). O questionário é autoaplicável, validado e específico para indivíduos com ELA, sendo composto por 40 questôes subdivididas em cinco domínios, com itens específicos para cada um. O objetivo desse questionário é mostrar a extensão do estado da doença em cada um dos cinco domínios, ou seja, nos domínios: 1 - Mobilidade (10 itens), 2 - Atividades da Vida Diária - AVDs (10 itens), 3 - Alimentaçáo e Deglutição (3 itens), 4 - Comunicação (7 itens) e 5 - Aspectos Emocionais (10 itens). A pontuação da escala varia entre 0 e 100 dentro de cada domínio. Uma pontuação de 0 a 19 em um domínio significa que o paciente não apresenta dificuldade; 20 a 39, raramente apresenta dificuldade; 40 a 59 , às vezes apresenta dificuldade; 60 a 79, frequentemente apresenta dificuldade; e 80 a 100 sempre apresenta dificuldade. Dessa forma, o questionário informa que quanto mais próximo de zero, melhor a qualidade de vida e quanto mais próximo de cem, mais comprometida a qualidade de vida (PAVAN et al., 2007).

$\mathrm{Na}$ avaliação da independência funcional foi utilizada a Medida de Independência Funcional (MIF), por atender aos critérios de confiabilidade, validade, precisão e praticidade. É um instrumento de avaliaçâo da incapacidade de pacientes com restriçóes funcionais de origem variada, ou seja, tem como objetivo primordial avaliar de forma quantitativa a carga de cuidados demandada por uma pessoa para a realizaçáo de uma série de tarefas de vida diária. É composto por 18 itens que avaliam seis diferentes áreas (Cuidados Pessoais, Controle dos Esfíncteres, Mobilidade, Locomoção, Comunicação e Cognição Social), compreendendo itens motores e cognitivos, possuindo um sistema de graduaçáo da resposta do paciente que varia de 1 a 7 , sendo: 1 - dependência total; 2 - ajuda máxima (indivíduo realiza $>=25 \%$ da tarefa); 3 - ajuda moderada (indivíduo realiza $>=50 \%$ da tarefa); 4 - ajuda mínima (indivíduo realiza $>=75 \% \mathrm{da}$ tarefa); 5 - supervisão; 6 - independência modificada; e 7 - independência completa - assim, a pontuação total varia de 18 a 126 pontos (RIBERTO et al., 2004).

Nesta pesquisa foi utilizada a MIF exclusivamente com itens motores, dispensando-se a parte cognitiva desse protocolo e limitando-se o escore mínimo a 13 e o máximo a 91 pontos. Portanto, foram analisadas as quatro áreas motoras (Cuidados Pessoais, Controle de Esfíncteres, Mobilidade e Locomoção) e os respectivos 13 itens.

\subsection{Análise estatística}

Os dados obtidos foram tabulados e processados através do programa SPSS for Windows, versão 15.0. Inicialmente foi feita estatística descritiva visando caracterizar a amostra investigada por meio de médias, desvio padrão e frequências absolutas e relativas. $\mathrm{Na}$ sequência foi realizada estatística inferencial por meio do teste de correlação de Spearman para verificar a existência de relação entre o escore total da MIF motora e cada domínio do questionário ALSAQ-40/BR, e teste de Mann-Whitney para averiguar diferença entre indivíduos quanto ao escore da MIF motora e domínios da ALSAQ-40/ $\mathrm{BR}$, admitindo-se o nível de significância inferior a $5 \%(\mathrm{p}<0,05)$.

\section{Resultados}

A amostra analisada foi composta por 24 pacientes, $54,17 \%$ do sexo masculino e $45,83 \%$ do sexo feminino (Tabela 1). A média de idade encontrada foi de 58,6 \pm 11,74 anos, sendo 58,9 $\pm 11,43$ anos para os homens, e 58,2 $\pm 2,64$ anos para as mulheres. A média do tempo decorrido da doença era de $5 \pm$ 4,62 anos, sendo de 3,76 $\pm 3,11$ anos para homens e de $6,45 \pm 5,76$ para mulheres.

A MIF apresentou a média de 64,9 $\pm 20,55$ pontos no total, sendo $65,5 \pm 21,27$ para homens e $64,2 \pm 20,67$ para mulheres, indicando que todos os indivíduos pesquisados já apresentam dependência e necessitam de auxílio para realizar suas AVDs, 
porém sem diferença significativa entre os sexos e ausência de relação com as variáveis idade e tempo decorrido da doença $(\mathrm{p}>0,05)$.

Através do ALSAQ-40/BR verificou-se que todos os domínios estudados foram afetados (Tabela 2).

Os resultados da análise estatística mostraram que não houve correlação significativa entre esses domínios e a idade, bem como, entre eles e o tempo decorrido da doença $(\mathrm{p}>0,05)$.

Observou-se a existência de relação entre a MIF e os seguintes domínios da ALSAQ-40/BR: Mobilidade $(\mathrm{r}=-0,82 ; \mathrm{p}<0,01)$, AVDs $(\mathrm{r}=-0,67 ; \mathrm{p}<0,01)$, Alimentação $(r=-0,47 ; \mathrm{p}=0,02)$ e Comunicaçáo $(\mathrm{r}=-0,55 ; \mathrm{p}<0,01)$. Não foi encontrada relação significativa entre o domínio Aspecto Emocional e a $\operatorname{MIF}(r=-0,15 ; p=0,46)$ (Figura 1$)$.

\section{Discussão}

Doenças crônicas como a ELA produzem significativas repercussôes em diferentes esferas, como a econômica, social, emocional e familiar, afetando a $\mathrm{QV}$ dos pacientes como um todo. A doença, a perda de independência funcional e o estresse psicossocial possuem papéis importantes na percepção do paciente sobre a sua saúde ao longo da evoluçáo da doença (MELLO et al., 2009). Assim, o reconhecimento dos fatores que podem interferir na QV como, por exemplo, a dependência funcional dos indivíduos com ELA pode auxiliar na tomada de decisóes, na elaboraçáo de planejamentos e planos de intervenção, tratamento e serviços.

A deterioração física na doença varia extensamente entre os indivíduos ao longo do tempo (DAL BELLOHAAS; KLOOS; MITSUMOTO, 1998). E diante da evolução heterogênea inerente à ELA, é de se esperar que o comprometimento físico, a independência funcional e qualidade de vida também variem entre os pacientes. Portanto, a falta de correlação significativa entre a MIF e as variáveis idade e tempo decorrido de doença, bem como entre os domínios do ALSAQ-40/BR e as variáveis idade e tempo decorrido da doença em anos demonstram que uma menor independência funcional motora e qualidade de vida parecem não estar associadas com a idade ou tempo de doença.

O inexorável declínio da capacidade funcional com relativa preservação da cognição é percebido por muitos, inclusive os profissionais de saúde, como sendo o principal problema na vida dos pacientes. Devido a isto, frequentemente presume-se que aqueles com uma doença progressiva e incapacitante como a ELA são incapazes de ter prazer em viver e, invariavelmente, experimentam um declínio na

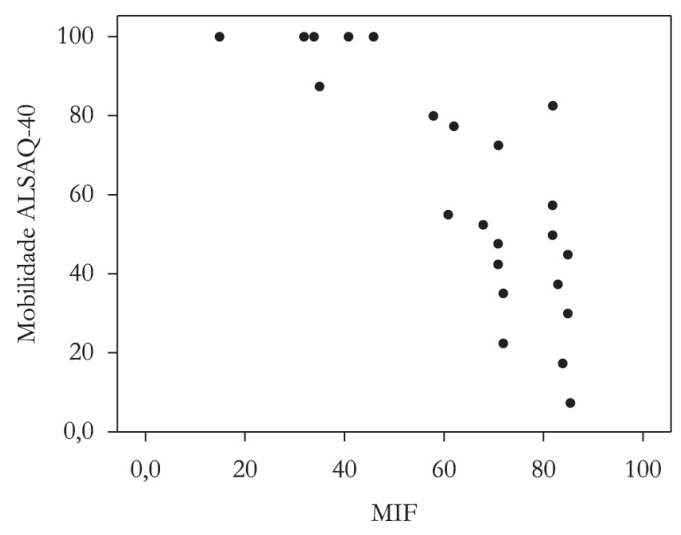

Figura 1. Correlação entre domínio Mobilidade do ALSAQ-40 e o escore da MIF.

Tabela 1. Distribuição da amostra por sexo, faixa etária, tempo de doença.

\begin{tabular}{cccc}
\hline & Masculino & Feminino & Total \\
\hline Amostra & 13 & 11 & 24 \\
Faixa etária & 34 a 79 anos & 39 a 79 anos & 34 a 79 anos \\
Tempo de doença & 1 a 10 anos & 1 a 15 anos & 1 a 15 anos \\
\hline
\end{tabular}

Tabela 2. Análise descritiva da distribuição dos escores da ALSAQ-40/BR.

\begin{tabular}{ccc}
\hline ALSAQ-40/BR & Mediana & Quartil25-Quartil75 \\
\hline Mobilidade & 53,7 & $41,2-83,7$ \\
AVDs & 50 & $35,6-78,1$ \\
Alimentação & 33,3 & $8,3-70,8$ \\
Comunicação & 44,4 & $24,1-93,7$ \\
Aspecto Emocional & 33,7 & $19,3-54,3$ \\
\hline
\end{tabular}

ALSAQ-40/BR: Amyotrophic Lateral Sclerosis Assessment Questionnaire; AVDs: Atividades de Vida Diária. 
sua qualidade de vida (HARDIMAN; HICKEY; O'DONERTY, 2004)

Através dos resultados do questionário ALSAQ-40/ $B R$ verificou-se que todos os domínios são afetados no curso dessa doença sendo o Aspecto Emocional o menos afetado.

A depressão é esperada na maioria dos pacientes com ELA, devido a fatores como tempo médio de sobrevida, deterioração da função motora (BOURKE; SHAW; GIBSON, 2001). Porém, contraditoriamente, em estudo realizado comparando cuidadores e pacientes quanto à qualidade de vida, ansiedade e depressão foi observado que nenhum dos grupos apresentou níveis de depressão (GILLIN, 1998). Outro estudo confirma que a depressão severa é rara em pacientes com ELA (EISEN; WEBER, 1999).

No entanto, apesar de não ter sido utilizada nenhuma escala específica para a depressão no presente estudo, o domínio Aspecto Emocional da ALSAQ-40/BR contempla questôes acerca dos sentimentos e da depressáo, e, portanto, diante dos estudos citados, espera-se que os escores no Aspecto Emocional sejam melhores que os de função motora.

A ELA ocasiona vários graus de dependência motora para realização das atividades diárias dos indivíduos devido ao comprometimento motor progressivo da doença (RODRIGUES et al., 2002). Um estudo transversal de 2008 encontrou todos os domínios da ALSAQ-40/BR afetados no curso dessa doença, sendo o domínio Mobilidade o mais afetado, corroborando esta pesquisa. $\mathrm{O}$ autor abordou que isso ocasiona impacto na qualidade de vida dos pacientes e influencia negativamente suas atividades diárias, agravadas pela dependência decorrente do comprometimento motor imposto pelo avanço da doença (BANDEIRA et al., 2010). De acordo com essas premissas, o presente estudo mostrou que a forte correlação inversa entre a MIF e os domínios Mobilidade e AVDs do ALSAQ-40/BR indica que quanto maiores são as dificuldades para mobilidade $\mathrm{e}$ realizaçáo de AVDs, menor o grau de independência funcional.

A ELA provoca disfagia e disartria na maior parte dos indivíduos afetados. Esses distúrbios são decorrentes da desintegração dos movimentos automáticos e voluntários que causam alteraçóes motoras na deglutição, fala e voz (fonoarticulação), manifestando-se por dificuldade em articular alguns sons e na fala, mastigação e deglutição, ocasionando engasgos frequentes (PIEMONTE, 2001; PONTES et al., 2010).

A comunicaçáo dos desejos, vontades e preferências do paciente à família e aos médicos é de vital importância e permite que o doente estabeleça relaçôes sociais com o meio que o cerca. Se há dificuldade na fala, o indivíduo terá problema para se expressar verbalmente e terá seu convívio social prejudicado. Sem comunicação, as suas necessidades podem ser incompreendidas (BOLMSJÖ, 2001) e a tomada de decisões dificultada, pois fica incapaz de expressar o pensamento (HILLEL; MILLER, 1987).

Segundo Bandeira et al. (2010), os domínios Alimentação e Comunicação também foram afetados. É de se esperar, portanto, que indivíduos mais comprometidos apresentem maior dificuldade para falar, preparar, levar à boca, mastigar e engolir o alimento, tornando-se mais dependente para a realização da tarefa. Confirmando, assim, a forte correlação inversa entre a MIF e Alimentação e a MIF e Comunicação encontradas neste trabalho.

Como já citado, segundo a OMS, "qualidade de vida" se refere à percepção do indivíduo da sua posição na vida, dentro do seu contexto de cultura e sistema de valores e em relação estabelecida com o ambiente em que vive (MINAYO; MARTZ; BUSS, 2000; DINIZ, 2006). Trata-se de um conceito complexo que inter-relaciona meio ambiente com aspectos físicos, psicológicos, nível de independência, relações sociais e crenças pessoais. Quando algum desses aspectos é alterado, tais como na diminuição da independência funcional para realizar atividades básicas, a qualidade de vida diminui.

A falta de correlaçáo significativa entre a variável Aspecto Emocional e a MIF demonstra que uma menor independência funcional motora parece não estar associada a alteraçóes emocionais. Em um estudo longitudinal de 2003 foi utilizado o questionário ALSAQ-40/BR para verificar as mudanças no indivíduo no curso da ELA, aplicando-se esse questionário duas vezes, sendo a segunda aplicação três meses após a primeira avaliação (NORQUIST; FITZPATRICK; JENKINSON, 2003). Nesse estudo observou-se deterioração significativa em pouco tempo decorrido da doença, com piora nos escores dos domínios da ALSAQ-40/BR Mobilidade, AVDs, Alimentação e Comunicação, enquanto a mudança nos escores do Aspecto Emocional não foi táo significativa na segunda avaliação, quando comparada à primeira.

Outro estudo, longitudinal, com o objetivo de avaliar como a ELA afeta a QV em diferentes domínios utilizando outros instrumentos, viu-se que os domínios de Saúde Mental e aspectos emocionais foram similares ao da população geral. $\mathrm{O}$ estudo demonstrou que apesar da deterioração física, a saúde mental dos pacientes permaneceu relativamente estável (DE GROOT et al., 2007). 


\section{Conclusão}

No presente estudo, viu-se que apesar do número amostral reduzido e da progressão heterogênea inerente à doença, todos os pacientes apresentaram diminuição da sua independência funcional, necessitando de auxílio para a realização de suas AVDs, bem como tiveram todos os domínios na qualidade de vida afetados, principalmente na área motora. No tocante aos resultados encontrados no domínio Aspecto Emocional do ALSAQ-40/BR, sugere-se uma limitação do instrumento, que apesar de específico para pacientes com ELA talvez não seja tâo sensível para captar tal informação.

Diante dos resultados, conclui-se que o uso desses instrumentos pode ser uma ferramenta de apoio na elaboraçáo de planos de intervenção e tratamento interdisciplinar, à medida que fornece informaçôes relevantes aos diversos profissionais $\mathrm{da}$ saúde. Determinar como a capacidade funcional causa impacto na QV dos pacientes pode auxiliar na atuação terapêutica mais personalizada e contribuir com o retardo da dependência funcional, com melhora da qualidade de vida das pessoas acometidas por esclerose lateral amiotrófica.

\section{Referências}

BANDEIRA, F. M. et al. Avaliação da qualidade de vida de pacientes portadores de Esclerose Lateral Amiotrófica (ELA) em Brasília. Revista de Neurociências, São Paulo, v. 18, n. 2, p. 133-138, 2010.

BOLMSJÖ, I. Existential issues in palliative care: interviews of patients with amyotrophic lateral sclerosis. Journal of Palliative Medicine, New York, v. 4, n. 4, p. 499-505, 2001. PMid:11798482. http://dx.doi. org/10.1089/109662101753381647

BOURKE, S. C.; SHAW, P. J.; GIBSON, G. J. Respiratory function vs sleep-disordered breathing as predictors of QOL in ALS. Neurology, Minneapolis, v. 57, n. 11, p. 20402044, 2001. http://dx.doi.org/10.1212/WNL.57.11.2040 BROOKS, B. R. et al. World Federation of Neurology Research Group on Motor Neuron Diseases. El Escorial revisited: revised criteria for the diagnosis of Amyotrophic Lateral Sclerosis. Amyotrophic Lateral Sclerosis and Other Motor Neuron Disorders, Estocolmo, v. 1, n. 5, p. 293-299, 2000.

CESÁRIO, C. M. M.; PENASSO, P.; OLIVEIRA, A. P. R. Impacto da disfunçáo motora na qualidade de vida em pacientes com acidente vascular encefálico. Revista de Neurociências, São Paulo, v. 14, n. 1, p. 6-9, 2006.

DAL BELLO-HAAS, V.; KLOOS, A. D.; MITSUMOTO, $\mathrm{H}$. Physical therapy for a patient through six stages of amyotrophic lateral sclerosis. Physical Therapy, Alexandria, v. 78, n. 12, p. 1312-1323, 1998.
DE GROOT, I. J. et al. Cross-sectional and longitudinal correlations between disease progression and different health-related quality of life domains in persons with amyotrophic lateral sclerosis. Amyotrophic Lateral Sclerosis and Other Motor Neuron Disorders, Estocolmo, v. 8, n. 6, p. 356-361, 2007.

DINIZ, D. P. Guias de medicina ambulatorial e hospitalar UNIFESP. São Paulo: Manole, 2006.

DURÁN, M. A. Fisioterapia motora na esclerose lateral amiotrófica. Revista de Neurociências, Sáo Paulo, v. 14, n. 2, p. 65-69, 2006.

EISEN, A.; WEBER, M. Treatment of Amyotrophic Lateral Sclerosis. Drugs Aging, New York, v. 14, n. 3, p. 173-196, 1999. http://dx.doi. org/10.2165/00002512-199914030-00003

FLECK, M. P. A. et al. A avaliaçáo de qualidade de vida: guia para profissionais de saúde. Porto Alegre: Artmed, 2008.

GILLIN, J. C. Are sleep disturbances risk factors for anxiety, depressive and addictive disorders? Acta Psychiatrica Scandinavica, Malden, v. 393, p. 39-43, 1998. Suplemento s393.

HAMILTON, B. B. et al. Performance profiles of the functional independence measure. American Journal of Physical Medicine \& Rehabilitation, Indianapolis, v. 72, n. 2, p. 84-89, 1993.

HARDIMAN, O.; HICKEY, A.; O'DONERTY, L. J. Physical decline and quality of life in amyotrophic lateral sclerosis. Amyotrophic Lateral Sclerosis and Other Motor Neuron Disorders, Estocolmo, v. 5, n. 4, p. 230-234, 2004.

HILLEL, A. D.; MILLER, R. M. Management of bulbar symptoms in amyotrophic lateral sclerosis. Advances in Experimental Medicine and Biology, New York, v. 209, p. 201-221, 1987.

JENKINSON, C. et al. Development and validation of short measure of health status for individuals with Amyotrophic Lateral Sclerosis/motor neurone disease: the ALSAQ -40. Journal of Neurology, Heidelberg, v. 246, n. 3, p. III16-III21, 1999. http://dx.doi.org/10.1007/ BF03161085

MCNAUGHTON, H. K.; WEATHERALL, M.; MCPHERSON, K. M. Functional measures across neurologic disease states: analysis of factors in common. Archives Physical Medicine and Rehabilitation, Reston, v. 86, n. 11, p. 2184-2188, 2005.

MELLO, M. P. et al. O paciente oculto: qualidade de vida entre cuidadores e pacientes com diagnóstico de esclerose lateral amiotrófica. Revista Brasileira de Neurologia, Rio de Janeiro, v. 45, n. 4, p. 5-16, 2009.

MINAYO, M. C. S.; MARTZ, Z. M. A.; BUSS, P. M. Qualidade de vida e saúde: um debate necessário. Ciência \& Saúde Coletiva, Rio de Janeiro, v. 5, n. 1, p. 7-18, 2000.

MITCHELL, J. D.; BORASIO, G. D. Amyotrophic Lateral Sclerosis. Lancet, Filadélfia, v. 369, n. 9578, p. 2031-2041, 2007. 
NORDON, D. G.; ESPÓSITO, S. B. Atualização em Esclerose Lateral Amiotrófica. Revista da Faculdade de Ciências Médicas de Sorocaba, Sorocaba, v. 11, n. 2, p. 1-3, 2009.

NORQUIST, J. M.; FITZPATRICK, R.; JENKINSON, C. Rash measurement in the assessment of amyotrophic lateral sclerosis patients. Journal of Applied Measurement, Maple Grove, v. 4, n. 3, p. 249-257, 2003.

PAVAN, K. et al. Adaptação transcultural do questionário de avaliação da esclerose lateral amiotrófica (ALSAQ-40) na cultura e linguagem brasileira. Medicina de Reabilitação, São Paulo, v. 26, n. 3, p. 73-76, 2007.

PIEMONTE, M. E. P. Manual de exercícios domiciliares para pacientes com ELA. São Paulo: Manole, 2001.
PONTES, R. T. Alteraçóes da fonação e deglutição na esclerose lateral amiotrófica: revisão de literatura. Revista de Neurociências, São Paulo, v. 18, n. 1, p. 69-73, 2010.

RIBERTO, M. et al. Validação da versão brasileira da medida de independência funcional. Acta Fisiátrica, São Paulo, v. 11, n. 2, p. 72-76, 2004.

RODRIGUES, G. et al. Avaliação sociológica de doentes com esclerose lateral amiotrófica. Revista Portuguesa de Pneumologia, Lisboa, v. 8, n. 6, p. 645-653, 2002.

VAN DEN BERG, J. P. Multidisciplinary ALS care improves quality of life in patients with ALS. Neurology, Minneapolis, v. 65, n. 8, p. 1264-1267, 2005. PMid:16247055. http://dx.doi.org/10.1212/01. wnl.0000180717.29273.12

\section{Contribuição dos Autores}

Nathalia Priscilla Oliveira Silva foi responsável pela elaboração do estudo, redação do texto, revisão e organização das fontes. Lizianne Juline do Nascimento e Silva Martins e Thaiana Barbosa Ferreira colaboraram na coleta dos dados da pesquisa e revisão do texto. Fabrícia Azevedo da Costa Cavalcanti foi a coordenadora da pesquisa e responsável pela revisão do manuscrito. 